\title{
Measurements of Reflected Shock Tunnel Freestream Nitric Oxide Temperatures and Partial Pressure
}

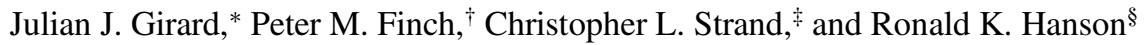 \\ Stanford University, Stanford, California 94305 \\ and \\ Wesley M. Yu,I Joanna M. Austin,,, and Hans G. Hornung主 \\ California Institute of Technology, Pasadena, California 91125
}

https://doi.org/10.2514/1.J060596

\begin{abstract}
This paper reports on measurements of freestream nitric oxide (NO) rotational and vibrational temperatures and partial pressures, collected in the Caltech T5 reflected shock tunnel. Quantum cascade lasers, emitting mid-infrared light resonant with fundamental rovibrational NO transitions, were directed through the supersonic (Mach $\sim 5$ ) freestream flow. Tunable diode laser absorption spectroscopy (TDLAS) was used to measure the path-averaged rotational and vibrational temperatures of NO in the flow, in addition to the NO partial pressure. The temperature measurements demonstrate strong evidence of NO rotational and vibrational equilibrium during the $1 \mathrm{~ms}$ test period. Agreement between vibrational and rotational temperatures was observed in all experiments, including one $\boldsymbol{h}_{\mathbf{0}, \infty} \approx$ $8 \mathrm{MJ} / \mathrm{kg}$ and four $h_{0, \infty} 18 \mathrm{MJ} / \mathrm{kg}$ experiments, during and after the nominal test time. Absorption from $\mathrm{CO}$ and $\mathrm{H}_{2} \mathrm{O}$ was also observed in the TDLAS measurements, though their concentrations cannot be accurately estimated. The goal of these and future experiments is to develop and demonstrate TDLAS experimental strategies for high-enthalpy impulse facilities and to help to inform improvements of existing models and solvers used for prediction of freestream conditions.
\end{abstract}

$\begin{array}{ll} & \text { Nomenclature } \\ A & =\text { integrated absorbance, } \mathrm{cm}^{-1} \\ A_{21} & =\text { Einstein } A, \mathrm{~s}^{-1} \\ c & =\text { speed of light, } \mathrm{cm} / \mathrm{s} \\ E & =\text { lower-state energy, } \mathrm{cm}^{-1} \\ g & =\text { degeneracy } \\ I & =\text { intensity, arbitrary units } \\ K_{B} & =\text { Boltzmann constant, } \mathrm{J} / \mathrm{K} \\ L & =\text { path length, cm } \\ P & =\text { pressure, atm or MPa } \\ Q & =\text { partition function } \\ S & =\text { linestrength, atm } \\ T & =\text { temperature, } \mathrm{K} \\ w & =\text { weights in fitting algorithm } \\ \alpha & =\text { absorbance } \\ \nu & =\text { frequency, } \mathrm{cm}^{-1} \\ \Phi & =\text { lineshape function, } \mathrm{cm}\end{array}$

Presented as Paper 2020-3714 at the AIAA Propulsion and Energy 2020 Forum, Virtual Event, August 24-28, 2020; received 29 January 2021; revision received 7 May 2021; accepted for publication 20 May 2021; published online 6 August 2021. Copyright ( 2021 by Julian Girard. Published by the American Institute of Aeronautics and Astronautics, Inc., with permission. All requests for copying and permission to reprint should be submitted to CCC at www. copyright.com; employ the eISSN 1533-385X to initiate your request. See also AIAA Rights and Permissions www.aiaa.org/randp.

*Graduate Student, Mechanical Engineering, Thermosciences Division, High Temperature Gasdynamics Laboratory, 452 Escondido Mall, Building 520;jjgirard@ stanford.edu. Student Member AIAA (Corresponding Author).

${ }^{\dagger}$ Graduate Student, Mechanical Engineering, Thermosciences Division, High Temperature Gasdynamics Laboratory, 452 Escondido Mall, Building 520. Student Member AIAA.

${ }^{\ddagger}$ Senior Research Engineer, Mechanical Engineering, Thermosciences Division, High Temperature Gasdynamics Laboratory, 452 Escondido Mall, Building 520. Member AIAA.

${ }^{\S}$ Clarence J. and Patricia R. Woodward Professor of Mechanical Engineering, Thermosciences Division, High Temperature Gasdynamics Laboratory, 452 Escondido Mall, Building 520. Fellow AIAA.

${ }^{\top}$ Graduate Student, Aerospace, Graduate Aerospace Laboratories, $1200 \mathrm{E}$. California Boulevard, MC 105-50. Student Member AIAA.

**Professor of Aerospace, Graduate Aerospace Laboratories, 1200 E. California Boulevard, MC 105-50. Associate Fellow AIAA.

${ }^{\dagger}$ C. L. "Kelly" Johnson Professor of Aeronautics, Emeritus, Graduate Aerospace Laboratories, 1200 E. California Boulevard, MC 105-50. Fellow AIAA.

\section{Introduction}

A T HYPERSONIC velocities, the onset of vibrational excitation, dissociation, and air chemistry have an impact on many parameters of interest to designers, including shock position, kinetic temperature, and heat loading [1 $\underline{-}-\underline{5}]$. Considerable recent effort has been devoted to understanding and modeling internal energy relaxation and dissociation in hypersonic flows using both first-principles calculations [6-9] and reduced-order modeling [10]. At present, the validation and implementation of models, ranging in fidelity and computational cost, is hampered by the lack of experimental data directly probing hypervelocity flowfields. Spectroscopic measurements in high-enthalpy, hypersonic experimental facilities thus continue to play an important role in grounding computational models and making them robust over a wide range of conditions [11-13].

Many experimental methods for characterizing flow facilities, such as heat flux gauges or pitot rakes, are intrusive and often yield incomplete information [14,15]. Optical techniques, such as schlieren imaging, provide nonintrusive metrics to comprehensively study the whole flow structure. More pointedly, spectroscopic measurements afford the possibility of species and state-specific information, which is critical in nonequilibrium environments. From this information, a variety of parameters can be discerned, such as temperature, composition, and flow velocity. It is desirable to leverage the wealth of spectroscopic knowledge to provide insight into hypersonic flow structures and nonequilibrium air. Unfortunately, the spectra of molecular oxygen and nitrogen pose significant challenges to experimental study. The strongest and widely known quantum transitions for these molecules are in the ultraviolet range, which are challenging to access due to the complexity and bulk of ultraviolet laser systems $[16,17]$. Transitions that are in more accessible spectral regions are quantum mechanically forbidden and are called "dark" [18,19].

Nitric oxide (NO) serves as an excellent tracer of nonequilibrium behavior of air for the following reasons: 1) NO forms as an intermediary in both the dissociation and recombination processes in air, and 2) NO has a well-understood and easily probed infrared absorption spectrum for the observation of vibrational and rotational energy. There is considerable heritage in using NO in hypersonic facilities. Danehy et al. used NO laser-induced fluorescence (LIF) for velocimetry in the Australian National University T2 and T3 free-piston shock tunnels [20]. Jiang et al. used NO planar LIF (PLIF) to investigate boundary-layer separation over models at the NASA Langley 31-Inch Mach 10 tunnel [21]. Lee et al. [22] and 
McMillin et al. [23] used PLIF of NO to study transverse fuel injection and combustion in supersonic flows in shock tube experiments at Stanford. Mohamed et al. used tunable infrared diode lasers for absorption measurements to probe NO concentrations, temperature, and velocity at the ONERA F4 wind tunnel [24].

In addition to measurements of flow over models at hypersonic conditions, there is also considerable interest in the facility freestream itself, particularly in reflected shock tunnels. Stagnation enthalpies and pressures are usually sufficient to induce chemical reactions and vibrational excitation. After the nozzle expansion, the flow may nominally approach a condition representative of a flight altitude and Mach number, but may retain a degree of thermal and chemical nonequilibrium [25]. This nonequilibrium may subsequently affect the flow structure in the presence of a body. NO originally formed in the stagnated region may persist longer within and downstream of the nozzle at concentrations that differ from thermochemical equilibrium projections and with multiple temperatures [26,27].

Parker et al. monitored NO concentration, temperature, and velocity of various hypersonic freestream shots at Calspan-University of Buffalo Research Center (CUBRC) in Buffalo, NY [28,29]. The authors used laser absorption spectroscopy with a quantum cascade laser (QCL) to probe several transitions near $5.45 \mu \mathrm{m}$ of NO and its isotopologues. Measurements were made in both the 48-in. tunnel and the Large Energy National Shock (LENS) I tunnel. By matching the integrated areas under the absorption features, the authors concluded that the NO concentration differed from computational fluid dynamics projections. Additionally, measurements of velocity were observed to substantially disagree with projections at high stagnation enthalpies (above $10 \mathrm{MJ} / \mathrm{kg}$ ), indicative of a lack of clarity of the "real gas" effects at play in the expanded flow. A study by Nompelis and Candler attempted to reproduce the results of Parker on a theoretical basis [30]. Distributing the total enthalpy into thermal, internal (mostly vibrational), and chemical modes, and then using theoretical kinetics for internal energy transfer and chemistry, the authors found that they could not account for Parker's lower measured NO levels (a discrepancy in mass fraction of about 4\%), nor the high level of energy consolidation in velocity at the exclusion of internal modes.

The present work is part of a Stanford University, California Institute of Technology, and University of Minnesota collaboration to characterize the Caltech T5 free piston shock tunnel. Optical diagnostics have been previously employed at the T5 facility $[31,32]$, but this study represents the first use of laser absorption on the shock tunnel. Tunable diode laser absorption spectroscopy (TDLAS) was employed to probe several rovibrational states of $\mathrm{NO}$, enabling measurement of population temperatures and species partial pressures. These state populations were analyzed to ascertain the temperatures of energy modes (i.e., rotational and vibrational) and investigate thermal nonequilibrium. This paper will discuss the TDLAS methodology used to infer freestream quantities of interest and preliminary measurements made at the T5 facility.

\section{Theory}

Freestream modal temperatures (rotational and vibrational) and molecular partial pressures in the T5 shock tunnel were measured through direct laser absorption. Specifically, scanned direct absorption (SDA) - by which a monochromatic laser is spectrally tuned across one or more molecular energy transitions of interest-was used to infer path-averaged freestream quantities. In general, this is achieved by directing laser light across the absorbing path length of interest (in this case the T5 freestream) and recording the fractional transmission of that light. Absorbance, defined as the negative log of fractional transmission, can be related to the relevant thermal and physical properties of the absorbing medium through the BeerLambert law [33], for transition $i$ and species $j$ :

$$
\begin{aligned}
\alpha_{i}(\nu) & =-\ln \left(\frac{I_{t}}{I_{0}}\right)_{\nu}=\int_{0}^{L} P_{j} S_{i}(T) \Phi_{i}(T, P, \nu) \mathrm{d} l \\
& =P_{j} L S_{i}(T) \Phi_{i}(T, P, \nu)
\end{aligned}
$$

If the path length is nonhomogenous, the pathwise distributions of the absorber partial pressure $P_{j}$, transition linestrength $S_{i}(T)$, and lineshape function $\Phi_{i}(T, P, \nu)$ are required to infer spatially resolved measures of pressure and temperature. If the path length is known and conditions can be assumed homogenous, the expression simplifies to a linear product as represented in Eq. (1). By spectrally tuning the laser light across a transition's absorbing linewidth, the measured absorbance can be frequency integrated to eliminate dependence on the lineshape function [Eq. (2)].

$$
A_{i}=\int_{-\infty}^{\infty} P_{j} L S_{i}(T) \Phi_{i}(T, P, \nu) \mathrm{d} \nu=P_{j} L S_{i}(T)
$$

The integrated absorbance $A$ is typically inferred by computationally fitting absorbance data to a representative lineshape model. In this study, the Voigt lineshape was chosen for its flexibility and accuracy at the measurement conditions observed. The Voigt lineshape model is represented by a two-parameter Gaussian-Lorentzian convolution that accounts for Doppler and pressure (or collisional) broadening.

Commonly, two-color thermometry is enabled by taking the ratio of integrated absorbance between two transitions of the target species, which is a function of temperature alone under the assumption of thermal equilibrium (i.e., $T=T_{\text {trans }}=T_{\text {rot }}=T_{\text {vib }}$ ). In this study, it was not assumed that a single temperature could define the energy distribution among absorber states. Sensitivity to multiple modal temperatures can be achieved by measuring several transitions originating from varying lower levels simultaneously. Specifically, in order to infer the rotational and vibrational temperatures independently, all simultaneous measures of NO state-specific absorbance were computationally fit to a nonequilibrium integrated absorbance model. This model is based upon the Beer-Lambert relation as expressed in Eq. (2), applying the theoretical formulation of linestrength as a function of the transition-specific Einstein $A$ coefficient state energies, and other molecular quantities as described by Hanson et al. [33].

$$
A_{i}=P_{j} L S_{i}(T)=L \frac{n_{1} \cdot A_{21, i} \cdot g_{2, i}}{8 \pi c \cdot \nu_{0, i}^{2} \cdot g_{1, i}}\left(1-\exp \left(\frac{-\nu_{0, i}}{K_{B} T}\right)\right)
$$

Assuming a Boltzmann population distribution, the model can be expressed as a function of the absorber number density or, by further assuming the ideal gas law as in Eq. (4), the absorber partial pressure:

$$
\begin{aligned}
A_{i}= & P_{j} L \frac{A_{21, i} \cdot g_{2, i}}{K_{B} T_{\operatorname{trans}} Q_{j}(T) \cdot 8 \pi c \cdot \nu_{0, i}^{2}}\left(\exp \left(\frac{-E_{1, i}}{K_{B} T}\right)\right) \\
& \left(1-\exp \left(\frac{-\nu_{0, i}}{K_{B} T}\right)\right)
\end{aligned}
$$

Here, the Einstein coefficient of spontaneous emission $A_{21, i}\left[\mathrm{~s}^{-1}\right]$, upper-state degeneracy $g_{2, i}$, lower-state energy $E_{1, i}$, and vacuum linecenter $\nu_{0, i}\left[\mathrm{~cm}^{-1}\right]$ are transition-specific parameters inferred directly from the most recent HITEMP database $[34,35]$. Note that a change in the units of pressure is needed for Eqs. (4) and (5); specifically pressure should be expressed in MPa instead of atm. Assuming that the rotational and vibrational energy distributions can be established independently with Boltzmann temperatures $T_{R}$ and $T_{V}$, respectively, and that translational energy remains in thermal equilibrium with rotational energy (i.e., $T_{\text {trans }}=T_{R}$ ), one arrives at the following expression:

$$
\begin{aligned}
A_{i}= & P_{j} L \frac{A_{21, i} \cdot g_{2, i}}{K_{B} T_{R} Q_{j}\left(T_{R}, T_{V}\right) \cdot 8 \pi c \cdot \nu_{0, i}^{2}}\left(\exp \left(\frac{-E_{R, 1, i}}{K_{B} T_{R}}+\frac{-E_{V, 1, i}}{K_{B} T_{V}}\right)\right) \\
& \left(1-\exp \left(\frac{-\Delta E_{R, i}}{K_{B} T_{R}}+\frac{-\Delta E_{V, i}}{K_{B} T_{V}}\right)\right)
\end{aligned}
$$

$E_{R, 1, i}$ and $E_{V, 1, i}$ are the rotational and vibrational lower-state energies, whereas $\Delta E_{R, i}$ and $\Delta E_{V, i}$ are the rotational and vibrational transition energy changes, respectively. 
Of special consideration in this model were the transition-specific parameters and total partition function $Q_{j}$ for NO. Though the NO HITEMP database accounts for certain hyperfine energy level splitting (i.e., magnetic interactions finer than $\Lambda$ doubling), at the conditions observed for this study, spectral line broadening made the corresponding transition separations unresolvable. Accordingly, certain steps were taken to avoid error in the interpretation of the spectral parameters. As expected from theory, $g_{2, i}$ was taken as $2 J+1$ (rotational level $J$ ). The total partition function was manually calculated, summing the contributions from all rotational and vibrational levels, accounting for spin-splitting and $\Lambda$ doubling, and allowing for distinct rotational and vibrational temperatures. All hyperfine transitions in HITEMP corresponding to a given vibrational, rotational, spin-split, and $\Lambda$-doubled level change (up to six) were grouped together and considered as a single rovibrational transition. The linecenter frequency of a transition grouping was taken as the mean of the individual frequencies weighted by their linestrengths, though this distinction would not significantly affect any of the measurements considering the small variance among hyperfine transition energies. The Einstein $A$ coefficient for the transition grouping was determined by summing the reference linestrengths $\left(T_{\text {ref }}=296 \mathrm{~K}\right.$ for HITRAN) and inferring the corresponding rate:

$$
A_{21, i}=\frac{S_{\text {ref }, i} \cdot Q_{j}\left(T_{\text {ref }}\right) \cdot 8 \pi c \cdot v_{0, i}^{2}}{\left[g_{2, i} \cdot \exp \left(-E_{1, i} / K_{B} T_{\text {ref }}\right)\right]\left[1-\exp \left(-\Delta E_{21, i} / K_{B} T_{\text {ref }}\right)\right]}
$$

A weighted least-squares approach was taken in computationally fitting the data to the absorbance model outlined in Eq. (5). At each time step, all $m$ concurrent integrated absorbance measurements were considered, weighting them by their corresponding measurement uncertainties. Removing this weighting scheme would impact the best-fit parameter values by less than $5 \%$. Equation (7) represents the relevant nonlinear minimization problem, where $A_{\text {model }, i}$ is the modeled nonequilibrium integrated absorbance of transition $i$ as given by Eq. (5), with $P_{\mathrm{NO}}, T_{R}$, and $T_{V}$ as free variables. $A_{\text {measured, } i}$ is the measured integrated absorbance inferred from the Voigt-fitted

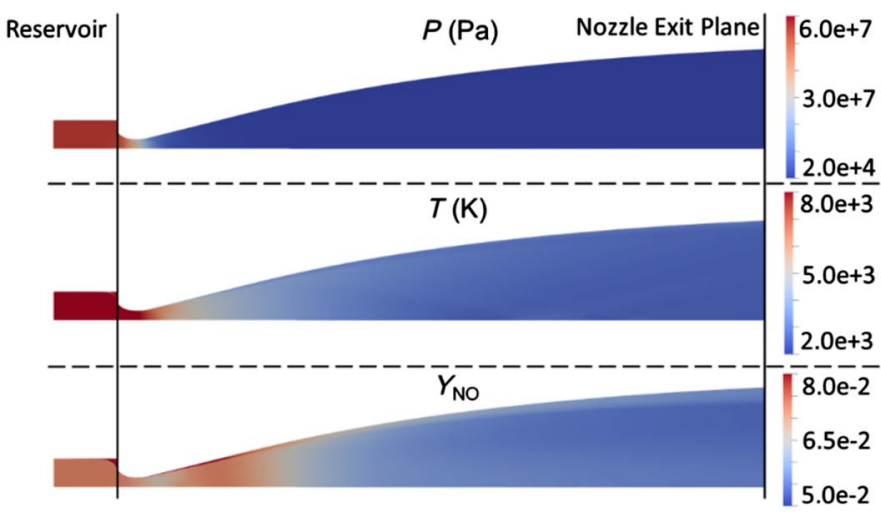

a)

Fig. 1 a) Axisymmetric Nozzle code simulations of total pressure, kinetic temperature, and NO mass fraction for the T5 nozzle at the conditions of shot 1 (see Fig. 2). b) Corresponding conditions at the exit plane as a function of nozzle radial position (radius $=15.7 \mathrm{~cm}$ ).

Table 1 Freestream and reservoir conditions for all T5 shots operated during this study (in order of operation)

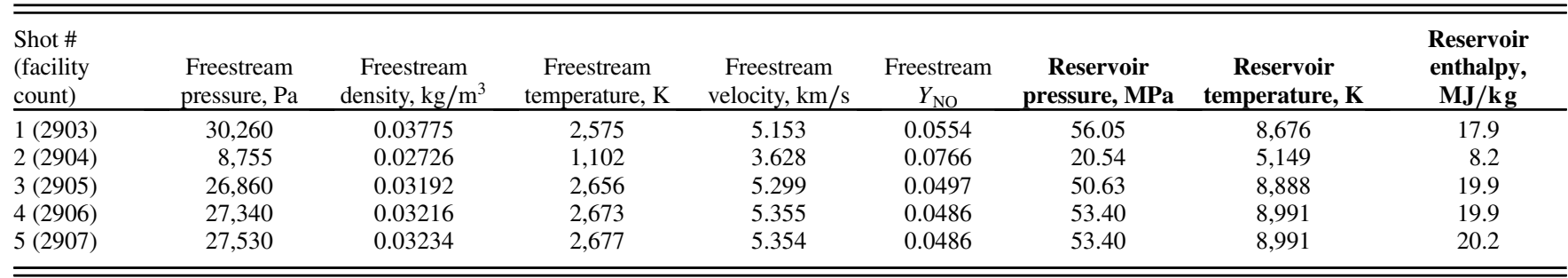

Reservoir conditions (bold) are inferred directly from measurements (with the aid of fundamental shock relations in the case of temperature and enthalpy), whereas the freestream conditions are simulated by the Nozzle code. absorbance data, and $w_{i}$ is the corresponding weighting factor based on the fit certainty:

$$
\left(\left[\begin{array}{c}
A_{\text {model }, 1}\left(P_{\mathrm{NO}}, T_{R}, T_{V}\right) \\
\vdots \\
A_{\text {model }, m}\left(P_{\mathrm{NO}}, T_{R}, T_{V}\right)
\end{array}\right]-\left[\begin{array}{c}
A_{\text {measured }, 1} \\
\vdots \\
A_{\text {measured }, m}
\end{array}\right]\right) \circ\left[\begin{array}{c}
w_{1} \\
\vdots \\
w_{m}
\end{array}\right]=\left[\begin{array}{c}
0 \\
\vdots \\
0
\end{array}\right]
$$

In practice, the inputs to this algorithm were the time-varying integrated absorbances for each measured transition and their corresponding standard errors. The outputs were the best-fit time-varying NO partial pressure, rotational temperature, and vibrational temperature, as well as the fit uncertainties of each. Species concentrations in this work are presented as partial pressures because the time-varying total flow pressure was not measured, and thus mole fractions could not be inferred accurately. The roughly homogenous core-flow diameter was taken as the effective absorbing path length, as discussed more detail in Sec. IV. The spectroscopic line-selection details, as well as notes on treatment of spectral interference are discussed in Sec. III.

\section{Spectroscopic Line Selection}

NO was selected as the spectroscopic target for the primary TLAS diagnostics employed in this study in consideration of its strong rovibrational absorption spectra and relatively high expected concentrations in the T5 freestream flow. Preliminary simulations(discussed in Sec. IV) - predicted NO mass fractions exceeding 5\% or the $18 \mathrm{MJ} / \mathrm{kg}$ total enthalpy condition and $7 \%$ for the $8 \mathrm{MJ} / \mathrm{kg}$ Stokes code discussed in Sec. IV) simulations, NO maintains a high equilibrium concentration within the hot reservoir immediately upstream of the nozzle and is chemically frozen during expansion (see Fig. 1), unable to reach the low concentration expected for equilibrated air at the freestream conditions.
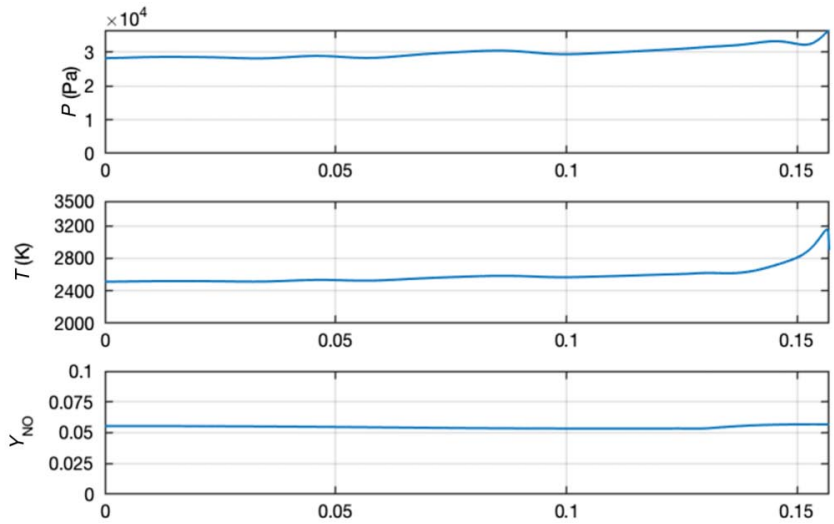

b) Nozzle Radial Position (m) (n)$$
\text { . }
$$ 
Targeting rovibrational absorption spectra with well-resolved transitions enabled high measurement sensitivity to the rotational and vibrational energy distributions in the freestream, and hence the corresponding modal temperatures. Specifically, the fundamental rovibrational band of $\mathrm{NO}$, which is active primarily in the range of 5-6 $\mu \mathrm{m}$ (see Fig. 2), was selected because of the high transition strength and isolation (low spectral blending) at the conditions of interest, and the prevalence of relatively cheap, compact infrared (IR) laser sources, detectors, optics, etc., in that spectral region. Formally, this rovibrational system is composed of overlapping fundamental bands $(\Delta v=1)$. Each observed individual transition within this system corresponds to a specific change in vibrational/rotational/ spin-split/ $\Lambda$-doubled/etc., level. At the conditions observed in this study, the frequency difference between paired $\Lambda$-doubled transitions could not be resolved (distinct peak separation) and were thus considered together. Accordingly, all NO transitions referred to in this study correspond to changes in spin-split rovibrational levels, without finer distinction.

Three lasers in total were used to probe the fundamental rovibrational bands of NO: one broadly tunable external cavity quantum cascade laser (EC-QCL) referred to here as laser 1 (numbering based on physical positioning during experimentation; see Fig. 3), and two distributed feedback quantum cascade lasers (DFB QCL), lasers 2 and 4 . The total tuning ranges of these lasers are indicated by the highlighted regions in Fig. 2.

The EC-QCL, from Daylight Solutions, had a large tunable range but relatively small rapid-scanning bandwidth (scan depth); only one transition could be sampled via current-injection tuning at a rate of $10 \mathrm{kHz}$. The laser was operated at a setpoint targeting $R_{2}(16.5)$ of the ground fundamental band (i.e., $R$-branch, $v^{\prime \prime}=0, J^{\prime \prime}=16.5$,
$\Omega=1.5$ ); see Table 2 . Unfortunately, mode-hop issues compromised data quality for this diagnostic in all but one experiment, the single shot run (shot 2) at the lower enthalpy condition.

Lasers 2 and 4, both DFB QCLs, were capable of significantly larger scan depths at the same tuning rate, enabling simultaneous measurement of many transitions during a single experiment. Two scan setpoints were used for laser 2, one targeting the spectral range $\sim 1900-1901 \mathrm{~cm}^{-1}$ (applied in all experiments but one of the higher enthalpy shots) and the second targeting $\sim 1897-1898.5 \mathrm{~cm}^{-1}$. The first setpoint of laser 2 provided measurements of several NO transitions, covering lower vibrational levels 0 through 3 , and lower rotational levels spanning $J^{\prime \prime}=6.5$ to $J^{\prime \prime}=43.5$ (see Table 2). The second setpoint of laser 2 also yielded several NO transition measurements, spanning the same lower vibrational levels and lower rotational levels $J^{\prime \prime}=5.5$ to $J^{\prime \prime}=42.5$, in addition to some unexpected $\mathrm{CO}$ and $\mathrm{H}_{2} \mathrm{O}$ transitions (discussed further in Sec. IV). Laser 4 was operated under one setpoint, which provided measurements of four NO transitions, together spanning the first two lower vibrational levels and lower rotational levels $J^{\prime \prime}=40.5$ to $J^{\prime \prime}=65.5$.

In some experiments, unexpected spectral interference from $\mathrm{CO}$ and $\mathrm{H}_{2} \mathrm{O}$ compromised measurements of $\mathrm{NO}$ transitions collected with laser 2. Specifically, $R_{1}(16.5)$ of the vibrational fundamental band from $v^{\prime \prime}=1$ (i.e., $R$-branch, $J^{\prime \prime}=16.5, \Omega=0.5$ ), measured with the first setpoint of laser 2 , overlapped significantly with a $\mathrm{CO}$ transition [ $\mathrm{P}(52)$ of the ground level fundamental band], observable in the bottom left plot of Fig. 2. The contribution of the $\mathrm{CO}$ transition to the integrated absorbance of the blended feature was subtracted in postprocessing, as described below. Measurements collected with the second setpoint of laser 2 included two resolvable $\mathrm{CO}$ transitions and a strong $\mathrm{H}_{2} \mathrm{O}$ transition.

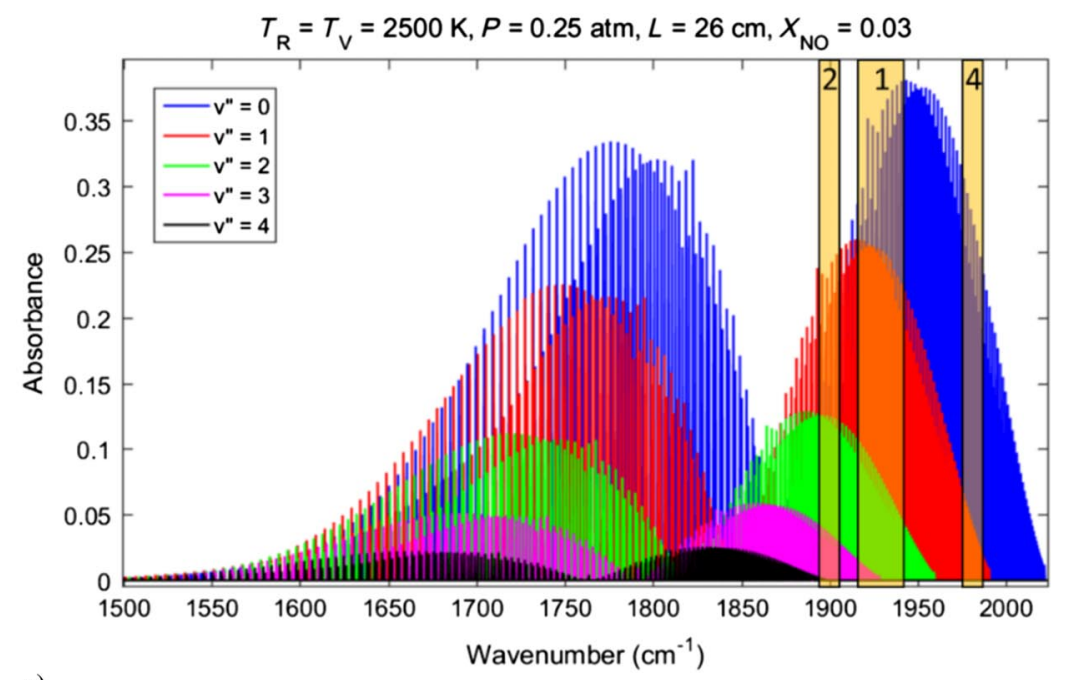

a)
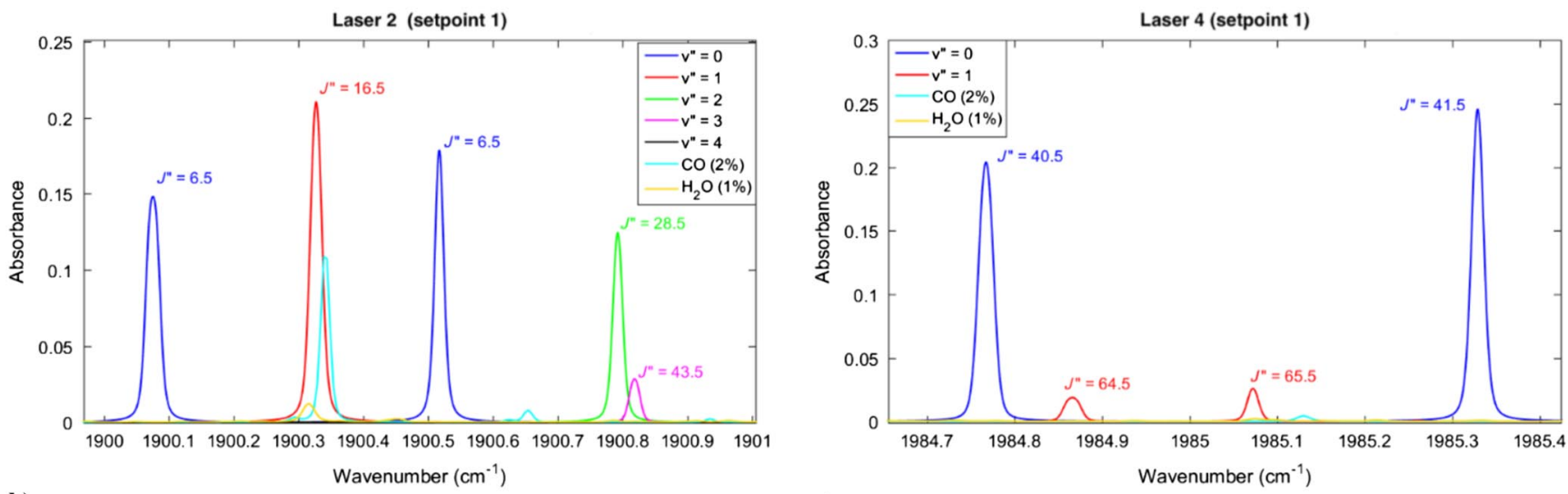

b)

c)

Fig. 2 a) Simulated NO fundamental band absorbance at conditions of a higher enthalpy shot. Shaded bar regions represent the tuning ranges of the lasers used in this study (see Table $\underline{2}$ and Fig. $\underline{3}$ ). b, c) Simulated absorbance across the individual rapid-scanning ranges of laser 2 (b) and laser 4 (c). 


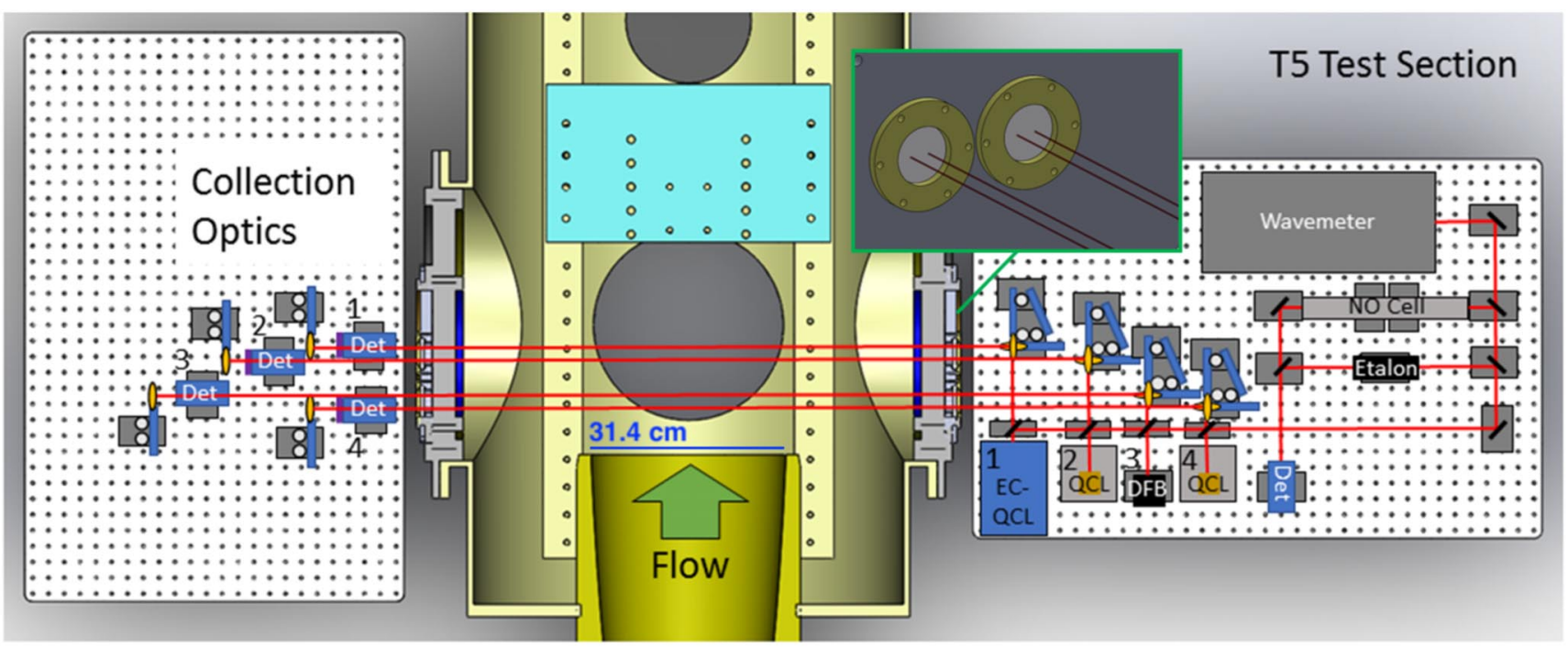

Fig. 3 Schematic of $\mathrm{T} 5$ facility measurement scheme. Light from four lasers (right side) was simultaneously directed in pairs through two sets of $50.8 \mathrm{~mm}$ $\mathrm{CaF}_{2}$ windows (see green-outlined box) and collected by four high-bandwidth photovoltaic detectors on the test section south side (left).

Table 2 All rovibrational transitions that contributed to measurements

\begin{tabular}{|c|c|c|c|c|c|c|}
\hline \multirow[b]{2}{*}{ Species } & \multirow[b]{2}{*}{ Vibrational level change } & \multicolumn{4}{|c|}{ Rovibrational transitions } & \multirow[b]{2}{*}{ Wavenumber range, $\mathrm{cm}^{-}$} \\
\hline & & Laser 1 & Laser 2, setpoint 1 & Laser 2, setpoint 2 & Laser 4 & \\
\hline \multirow[t]{4}{*}{$\mathrm{NO}$} & $0 \rightarrow 1$ & $R_{2}(16.5)$ & $R_{1}(6.5), R_{2}(6.5)$ & $R_{2}(5.5)$ & $R_{2}(40.5), R_{1}(41.5)$ & $1896.98-1985.33$ \\
\hline & $1 \rightarrow 2$ & & $R_{1}(16.5)$ & $R_{1}(15.5)$ & $R_{2}(64.5), R_{1}(65.5)$ & $1897.63-1985.07$ \\
\hline & $2 \rightarrow 3$ & & $R_{1}(28.5)$ & $R_{2}(26.5)$ & & $1897.51-1900.79$ \\
\hline & $3 \rightarrow 4$ & & $R_{2}(43.5)$ & $R_{2}(41.5), R_{1}(42.5)$ & & 1897.64-1900.82 \\
\hline $\mathrm{H}_{2} \mathrm{O}$ & $v_{2}: 0 \rightarrow 1\left(v_{1}, v_{3}=0\right)$ & & {$[J: 13 \rightarrow 14$} & $K_{a}: 1 \rightarrow 2, K_{b}: 12 \rightarrow 1$ & & 1897.52 \\
\hline \multirow[t]{2}{*}{$\mathrm{CO}$} & $2 \rightarrow 3$ & & & $P(43)$ & & 1898.39 \\
\hline & $4 \rightarrow 5$ & & & $P(33)$ & & 1897.89 \\
\hline
\end{tabular}

The convention used for NO and CO rovibrational transitions is $\operatorname{Branch}_{Z}\left(J^{\prime \prime}\right)$, where $Z$ defines the spin-splitting quantum number $\Omega$ (i.e., 0.5 and 1.5 for $Z=1$ and $Z=2$, respectively). All $\mathrm{H}_{2} \mathrm{O}$ and $\mathrm{CO}$ transitions were measured with laser 2, operating under setpoint 2.

An iterative processing methodology was developed to account for unexpected $\mathrm{CO}$ absorption interference (encountered with the first setpoint of laser 2) so that the NO absorbance contribution to a blended feature could be estimated. First, the data from laser 2 operated under its second setpoint were processed to estimate the time-varying $\mathrm{CO}$ partial pressure evaluated at the measured NO temperature for that experiment. Under the assumption that the $\mathrm{CO}$ partial pressure was roughly consistent among the higher enthalpy shots, the $\mathrm{CO}$ absorbance contributing to the blended transition was inferred by evaluating at the first iteration estimate of NO temperature. With $\mathrm{CO}$ subtracted from the blended $\mathrm{CO}-\mathrm{NO}$ feature, the NO contribution could be input to the next iteration of the temperature measurement. Typically, a few iterations were necessary for the measured temperature to converge. Similarly, measurement of the $\mathrm{H}_{2} \mathrm{O}$ transition accessed with the second setpoint of laser 2 required subtraction of a smaller interfering NO transition. The integrated absorbance of the NO transition was simulated, evaluating at the temperature and partial pressure measured with unimpacted transitions for that experiment, and subsequently subtracted from the blended $\mathrm{NO}-\mathrm{H}_{2} \mathrm{O}$ feature. In several other cases, transitions were blended to the extent that two or more had to be fit simultaneously, with an ultimately reduced fit certainty and a corresponding effect on the inferred temperature and partial pressures. Though these subtractions and special fitting treatments increased measurement error, the collection of measured transitions for each shot was still large enough to provide strong sensitivity to rotational and vibrational temperature, as well as NO partial pressure.

Out of a total of $17 \mathrm{NO}$ transitions sampled with lasers 1, 2, and 4 across all experiments, 8 required extra corrections or subtractions or were altogether omitted due to spectral interference (i.e., transition blending). Note that those transitions that were omitted from measurements are not included in Table 2. For three of these transitions the spectral interference was caused by $\mathrm{CO}$ and $\mathrm{H}_{2} \mathrm{O}$ absorption. In the other cases, blending of neighboring NO transitions was responsible. Despite significant errors associated with the corrections applied to many of the measured features (discussed in Sec. IV), the sheer number of measurements coupled with the wide range of accessed vibrational and rotational levels provided adequate sensitivity to the measures of interest, namely, rotational and vibrational temperatures and NO partial pressure. Future experiments will involve a careful line selection, to minimize undesired spectral interference.

\section{Experimental Details}

The measurements presented in this study were made at the T5 reflected-shock tunnel facility, at the Graduate Aerospace Laboratories at the California Institute of Technology (GALCIT). T5 can achieve reservoir conditions of $25 \mathrm{MJ} / \mathrm{kg}$ total enthalpy and test times on the order of 1-2 ms in duration (though test times were closer to $1 \mathrm{~ms}$ in this study) [36]. During operation, a piston is loaded into one end of a compression tube filled with a driver gas. A secondary reservoir pressurizes the volume behind the piston, accelerating it into the driver gas to speeds in excess of $300 \mathrm{~m} / \mathrm{s}$. When a steel diaphragm ruptures, a shock wave is generated in the driven test gas (air, in these experiments). The shock wave propagates through the shock tube, then reflects from the end wall, and the twice-shocked test gas (at temperatures and pressures up to $10,000 \mathrm{~K}$ and $100 \mathrm{MPa}$ ) serves as the reservoir supply for the Mach 6 contoured nozzle used in this study. 
The reflected shock propagates back upstream through the shock tube and is eventually incident on the downstream-propagating interface between the expanded driver gas and postshock test gas. A tailored condition is achieved when the reflected shock-contact surface interaction results in no reflected wave, such that the reservoir remains nominally stagnated. In an undertailored condition, a reflected expansion fan is generated and propagates back toward the nozzle throat. Although a tailored condition is ideal, in practice three-dimensional and viscous effects limit the uniform cancellation of the reflected wave. An undertailored condition can be selected to maximize the test time by delaying the arrival of the driver gas. Test time begins when the nozzle flow is established after the startup transient (typically $1 \mathrm{~ms}$ after the incident shock arrival at the reservoir pressure gauge, $t=0 \mathrm{~ms}$ ) and is terminated by the arrival of driver gas contamination. For these experiments, TDLAS data were collected for much longer than the test time; the first $4 \mathrm{~ms}$ of processed data are presented here.

Five shots in total were run for this study (see Table 1): four at a repeated $18 \mathrm{MJ} / \mathrm{kg}$ stagnation enthalpy condition (enabling assessment of the facility shot-to-shot consistency), and one at an $8 \mathrm{MJ} / \mathrm{kg}$ setpoint. Freestream conditions are calculated for each run using the experimental shock speed and postreflected shock reservoir pressure as inputs into axisymmetric, reacting Navier-Stokes simulations with a five-species air model [37-39], shown in Fig. 1a. The model assumes $T_{\text {trans }}=T_{\text {rot }} \neq T_{\text {vib }}$. The code was not designed to handle a time-evolving reservoir; instead the static reservoir conditions calculated from measured shock velocity and reservoir pressure are inputs to the code. Thus, in this work, only a static (i.e., constant in time) estimation of freestream conditions is reported for comparison. The simulations indicate a well-defined core flow with highly homogenous conditions at the nozzle exit (evident in the simulated exit plane conditions displayed in Fig. 1), supporting application of the path-averaged LAS measurement scheme [i.e., removal of the pathintegral from the absorbance model, Eq. (1)].

The sensor developed by Stanford for the T5 freestream measurements included four lasers in total (see Fig. 3). Three of the lasers were used to probe the NO fundamental rovibrational band, together spanning vibrational levels $0-3$, and rotational levels 5.5-65.5. Additionally, one laser was used to probe an excited atomic oxygen transition. Because of signal-to-noise ratio (SNR) limitations encountered with this laser, in addition to suspected drift in the tuning range, results from this diagnostic were inconclusive. All presented measurements were collected with the three QCLs targeting NO (lasers 1,2, and 4). These were current-injection-tuned at a frequency of $10 \mathrm{kHz}$ with a triangular input waveform (see Fig. 4), yielding an effective measurement rate of $20 \mathrm{kHz}$.

Laser 1 was an EC-QCL, from Daylight Solutions, with a typical scan depth of $\sim 0.2 \mathrm{~cm}^{-1}$. Though it was operated for all experiments, laser 1 only provided usable absorption data of the target transition [ $R_{2}$ (16.5); see Table 2] for the lower enthalpy shot and was otherwise susceptible to data-compromising mode-hops induced by physical vibrations (e.g., facility movement during the shot). Fortunately, none of the other lasers were subject to this difficulty. Laser 2, acquired from AdTech Optics, was a DFB-QCL with a tunable bandwidth centered near $1900 \mathrm{~cm}^{-1}$ and operated at two rapid-tuning setpoints, each with a scan depth of $\sim 1 \mathrm{~cm}^{-1}$. Laser 4 , a DFBQCL manufactured by Alpes Lasers and centered near $1985 \mathrm{~cm}^{-1}$, was operated at a single setting across all experiments, with a scan depth of $\sim 1 \mathrm{~cm}^{-1}$, which accessed 2-4 transitions.

For each experiment the lasers were directed in pairs through two sets of wedged 2-in. $\mathrm{CaF}_{2}$ windows (as displayed in Fig. 3). The measurements thus integrated along paths passing through the entire test section. The distance between the outer flanges of the test section where the windows were mounted was $\sim 76 \mathrm{~cm}$ (measured from the inside of the tunnel), but the effective core-flow diameter was only $25.9 \mathrm{~cm}$, as estimated by the Nozzle code. To mitigate the effect of facility vibrations on measurement SNR, special care was taken to structurally reinforce the entire optical assembly, both at the level of the supporting structure for the optical table, and the mounting optomechanics for individual lasers, detectors, mirrors, and filters. On the collection side of the test section, the four beams were focused by concave mirrors and passed through bandpass filters $(<500 \mathrm{~nm}$ Full-Width Half-Maximum (FWHM)) to eliminate potential shorterwavelength emission before being collected by high-bandwidth Vigo Systems photovoltaic (PV) detectors with $9 \mathrm{~mm}^{2}$ active areas. Detector signals were recorded by a 4-channel 12-bit NI DAQ, sampling at $10 \mathrm{MSa} / \mathrm{s}$.

\section{Results}

The raw data collected during the experiments described above, and as displayed in Fig. 4, were converted into integrated absorbance time histories by computationally fitting the transition absorbances to a Voigt lineshape model. It was observed that Voigt fitting of the raw absorbance data was optimal (supported by fit residuals) while varying Doppler-broadening linewidth, linecenter position, and integrated absorbance, and by fixing the temperature- and pressuredependent collisional broadening width at its expected value. The time-varying integrated absorbance for each transition was smoothed with a moving-average filter (quadratic regression with a span of $\sim 10-20 \%$ of the maximum number of measurement points for a given experiment) to mitigate the effects of spurious noise on the stability of the temperature measurements (see Fig. 5). In general, the measurement quality observed was notably high, indicated by the high SNR ( 40 at peak absorbance) of these absorbance measurements.
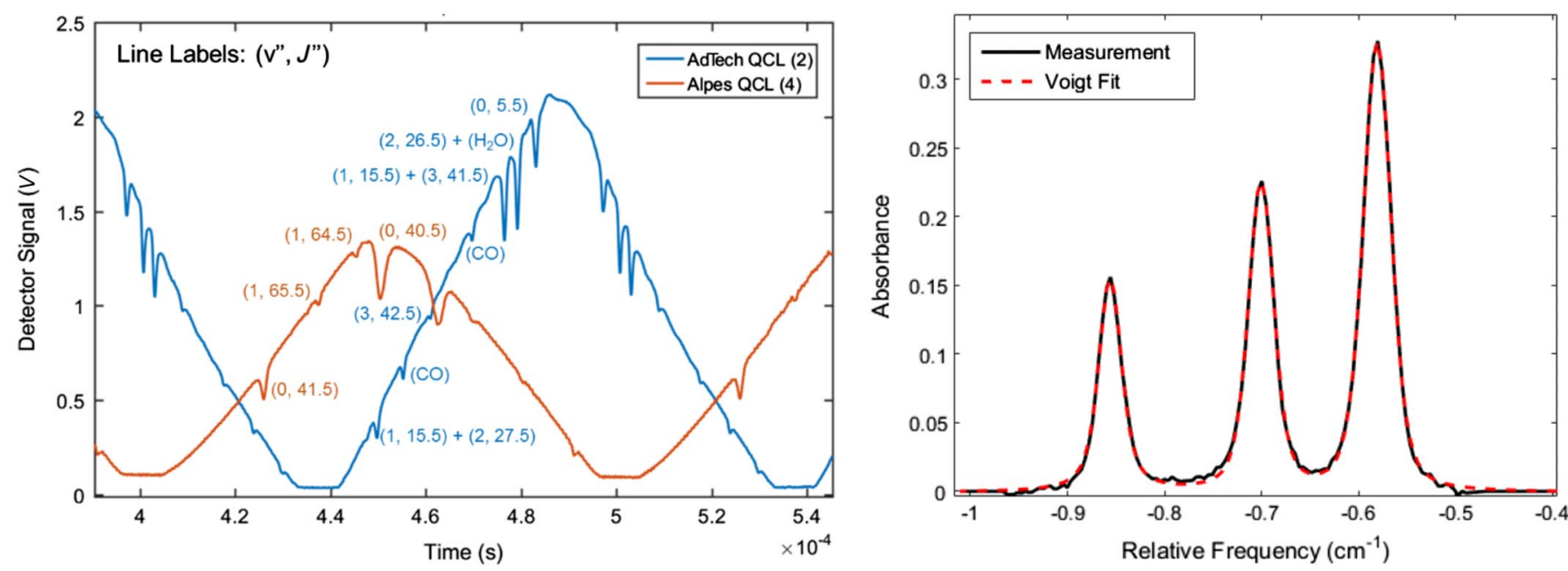

a)

b)

Fig. 4 a) Raw detector signal from the two QCL diagnostics, the AdTech laser (blue) and the Alpes laser (orange). b) Example absorbance trace for a cluster of transitions measured in the same experiment with best Voigt-fit overlaid. This data corresponds to higher enthalpy shot 4 (see Table 1 for details). 
Shot 1: $2575 K, 30260 \mathrm{~Pa}, 5.153 \mathrm{~km} / \mathrm{s}$ (Laser 2)

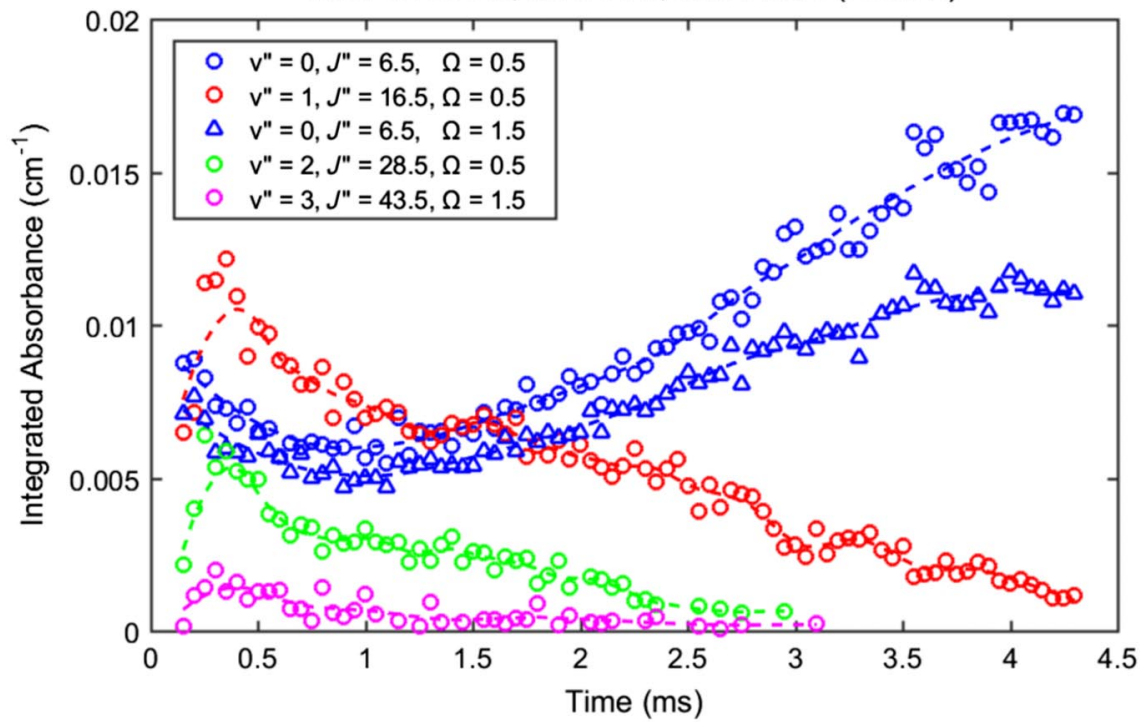

Fig. 5 Measured integrated absorbance time histories for transitions measured with the AdTech QCL (laser 2) during a higher enthalpy shot (shot 1; see Table 1). To mitigate the effect of spurious absorbance noise on temperature measurements, these data were smoothed with a moving-average filter (dashed lines).

As outlined in Sec. II, the measured time-varying integrated absorbance data for each transition were fit to the nonequilibrium absorbance model using a weighted least-squares approach. This model was based on the assumptions of a homogenous absorbing path length equal to the core-flow diameter (10.2 in.) and that the rotational and vibrational energy distributions were independent and Boltzmann with $T_{\text {trans }}=T_{\text {rot }} \neq T_{\text {vib. }}$. The solving routine was improved by weighting the impact of transitions according to their individual integrated absorbance uncertainties. Figure 6 displays the results for four out of the five total experiments, presented chronologically with corresponding conditions above each plot (appearing
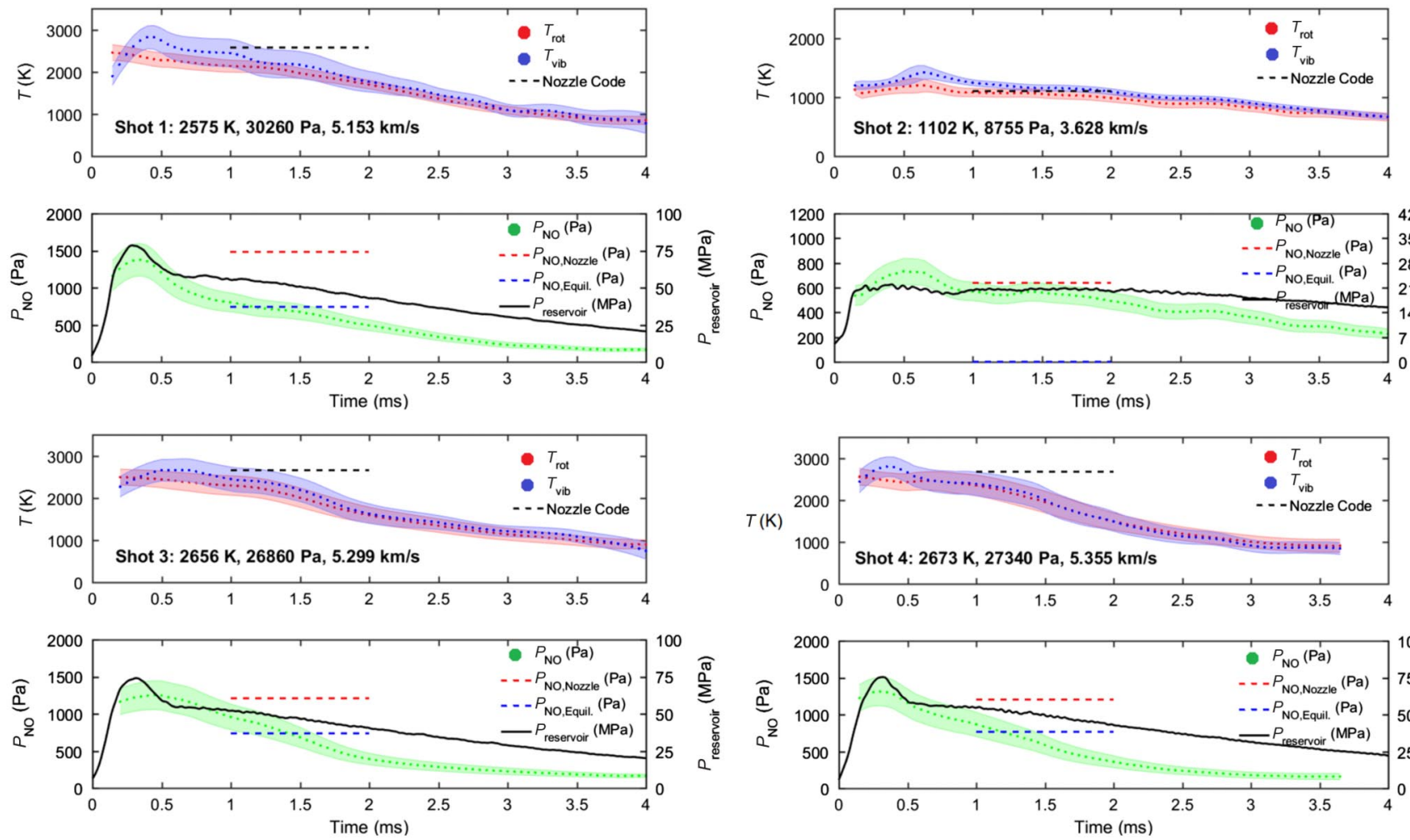

also in Table 1). The time-varying rotational and vibrational temperatures, as well as the partial pressure of NO, are all derived from the LAS measurements of NO. For comparison, the Nozzle code static prediction of temperature and $P_{\mathrm{NO}}$ during the approximate test time, as well as the equilibrium $P_{\mathrm{NO}}$ simulated at each condition with seven-species air (GRI-mech 3.0 [40]), and the measured reservoir pressure (mean of two sidewall transducers) are plotted. Qualitatively, the data indicate good agreement between the rotational and vibrational temperatures, particularly during and after the nominal test time $t(1-2 \mathrm{~ms})$. The higher enthalpy shots appear to follow a similar trend in decreasing temperature and NO partial pressure,
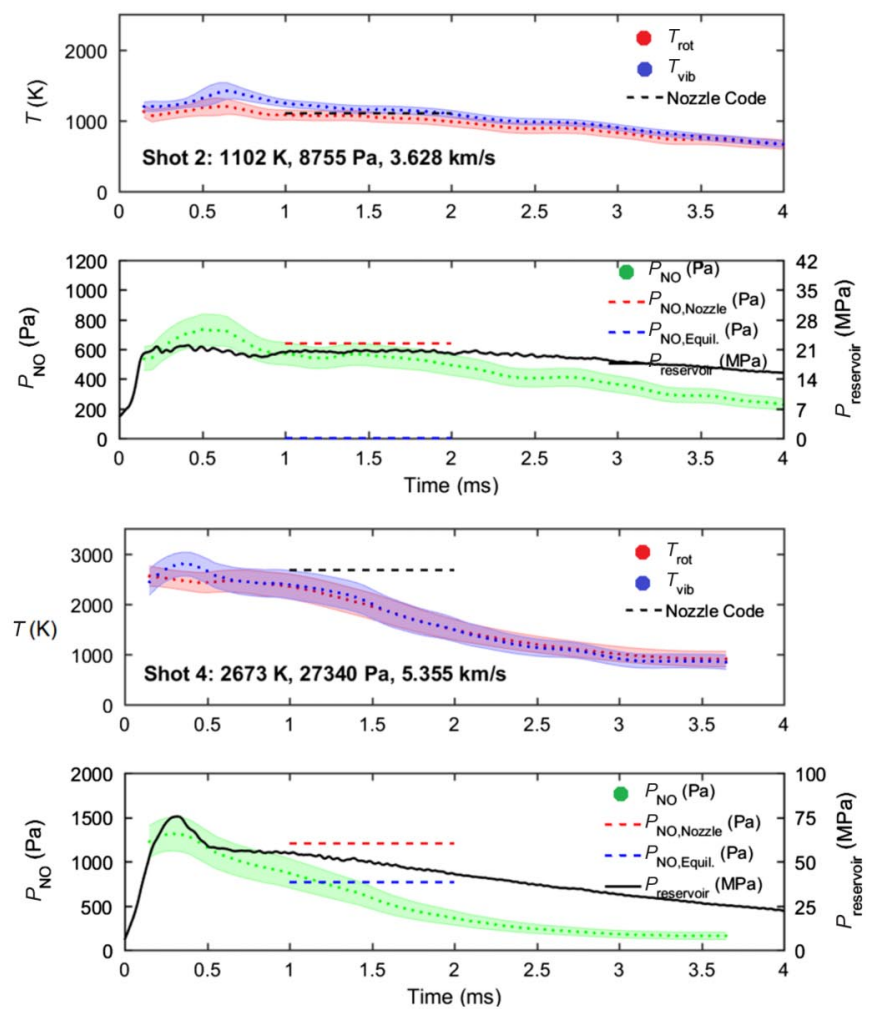

Fig. 6 Measured rotational and vibrational temperature and NO partial pressure during the first 4 ms of nozzle flow. Shaded regions indicate estimated 1- $\sigma$ uncertainty bounds on measurements. $P_{\mathrm{NO}, \text {,Equil }}$ represents the equilibrium partial pressure evaluated at the Nozzle code temperature using thermochemical data [ㅇ] (blue dash). Shot 5 results not shown. 
which is mirrored in the reservoir pressure trace as this condition was undertailored. Additionally, for all four of the higher enthalpy shots (shot 5 not shown in Fig. 6) the measured $P_{\text {NO }}$ during the test time agrees more closely with the equilibrium $P_{\mathrm{NO}}$ than the Nozzle code prediction. The lower enthalpy shot also suggests a slightly decreasing trend in temperature and NO partial pressure, but varies comparatively less. Furthermore, there is improved agreement with the Nozzle code at the lower enthalpy condition, possibly a result of a more closely tailored reservoir. It is unknown whether improved tailoring of the higher enthalpy shot condition would improve agreement of measurements with Nozzle code. To give a sense of the maximum contribution of NO to the freestream mixture composition, the approximate peak $P_{\mathrm{NO}}$ at the higher and lower enthalpy conditions were $\sim 1400$ and $\sim 700 \mathrm{~Pa}$, corresponding to mole fractions of $\sim 5$ and $\sim 8 \%$, respectively, evaluated at the Nozzle code prediction of total pressure.

In addition to the many NO transitions successfully measured, $\mathrm{H}_{2} \mathrm{O}$ and $\mathrm{CO}$ absorption was also observed. Though the presence of measurable quantities of these species was unexpected, it is not unprecedented in hypersonic facility freestream flows [24]. The integrated absorbance from the stronger of two resolvable $\overline{\mathrm{CO}}$ transitions (visible in Fig. 4) is plotted against time in Fig. 7. Unfortunately, without making strong assumptions about the temperature and spatial distribution of $\mathrm{CO}$ and $\mathrm{H}_{2} \mathrm{O}$ in the freestream, their concentrations cannot be estimated. The source of $\mathrm{CO}$ and $\mathrm{H}_{2} \mathrm{O}$ in the test section is not yet understood. Sources of carbon include the Mylar diaphragm (which is effectively vaporized shortly after reflection of the incident shock and passes through nozzle) and any residual hydrocarbon solvents used to clean the tubes between shots. Residual hydrocarbons and ambient water adsorption to the T5 walls could potentially account for the measured freestream concentrations of $\mathrm{H}_{2} \mathrm{O}$. Absorption from ambient $\mathrm{H}_{2} \mathrm{O}$ outside of the test section was observed, but was easily subtracted as a background artifact in both the transmitted and incident intensities [see Eq. (1)]. In future measurement campaigns, $\mathrm{CO}$ and $\mathrm{H}_{2} \mathrm{O}$ absorption spectra must be considered during the line-selection process, as interference from these species proved damaging to measurement quality for some NO transitions, as outlined in Sec. III. Ultimately, the observed levels of $\mathrm{CO}$ and $\mathrm{H}_{2} \mathrm{O}$ offer an opportunity to expand the number of spectroscopic targets measured in future freestream characterization experiments. A major goal of future measurement campaigns will be to quantitatively estimate the concentrations of $\mathrm{CO}$ and $\mathrm{H}_{2} \mathrm{O}$ in the uniform freestream core, so that the impact of such contaminants on hypersonic flow-model experiments can be evaluated.

Table 3 lists the primary sources of measurement error, and their magnitudes, for the results presented in this section. Magnitudes of error sources are presented as a percentage of the total measurement quantity. To simplify this error analysis, all sources were assumed to act on overall measurement error independently. The error in integrated absorbance $A$ is effectively the Voigt-model fit error, including interference-subtraction uncertainty when applicable. Its magnitude was estimated computationally via Monte Carlo simulation for the

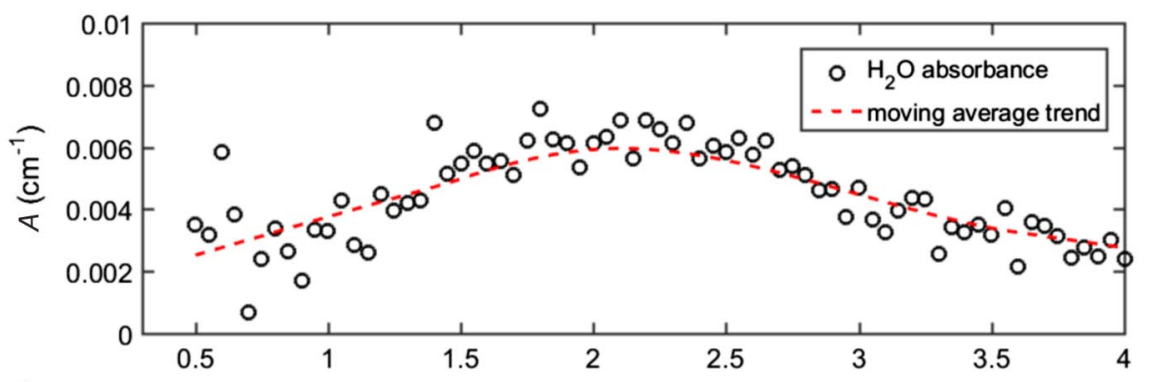

a)

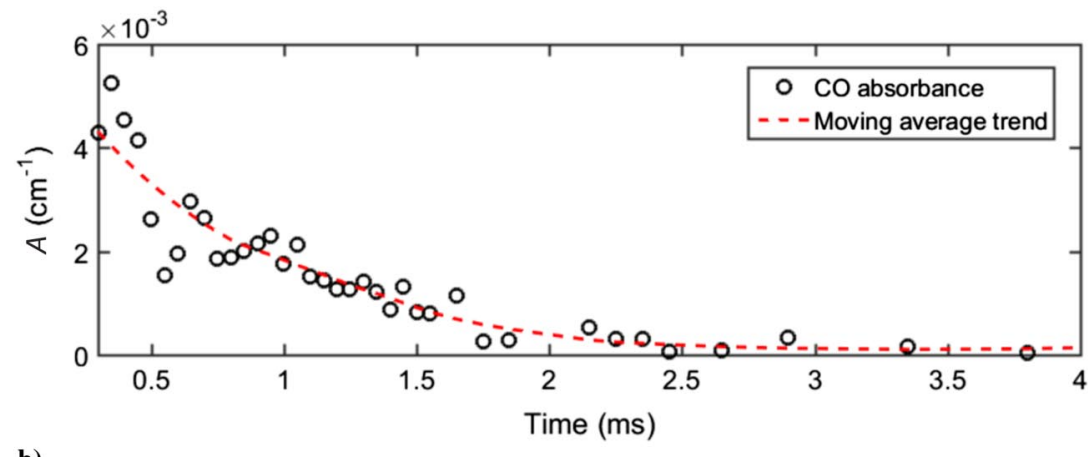

b)

Fig. 7 Integrated absorbance of $\mathrm{H}_{2} \mathrm{O}$ (a) and $\mathrm{CO}$ (b), both measured with the AdTech QCL (laser 2), operating under its second setpoint, for a higher enthalpy shot (shot 4; see Table 1).

Table 3 Uncertainty sources and their contributions to the typical total 1- $\sigma$ error for the presented measurements

\begin{tabular}{|c|c|c|c|c|c|c|}
\hline \multirow[b]{2}{*}{ Measurement } & \multicolumn{5}{|c|}{ Individual source contributions to total, $\%$} & \multirow[b]{2}{*}{ Typical total $1-\sigma$ error, $\%$} \\
\hline & $A$ (fits) & Spectral parameters & Absorbance model fit & Temperature & Path length & \\
\hline$T_{\text {rot }}$ & 6 & 1 & 1 & -- & -- & 8 \\
\hline$T_{\text {vib }}$ & 8 & 1 & 1 & -- & -- & 10 \\
\hline$P_{\mathrm{NO}}$ & 4 & 2 & 1 & -- & 9 & 16 \\
\hline$A_{\mathrm{H}_{2} \mathrm{O}}$ & 12 & -- & -- & -- & -- & 12 \\
\hline$A_{\mathrm{CO}}$ & 7 & -- & -- & -- & -- & 7 \\
\hline
\end{tabular}

For measurements based on integrated absorbances of NO transitions, the error assigned to $A$ includes the propagated uncertainty from all the NO transitions considered simultaneously in the absorbance model. 
NO measurements. Spectral parameter error is primarily the uncertainty in the inferred Einstein $A$ coefficient, but includes the error in all parameters taken from HITEMP. The absorbance model error, associated with the computational procedure for fitting the many measured NO transitions to temperatures and partial pressure, was relatively small for all experiments due to the high measurement sensitivity with respect to these measures. Absorbing path length error was conservatively estimated as $10 \%$ to accommodate for an absorbing boundary layer beyond the core flow, contributing to the error of all partial pressure measurements. This simplified error analysis indicates that considerable measurement error improvements could be made by eliminating the need for highly uncertain interference subtractions and blended feature fitting, in addition to limiting the absorbing path length to the homogenous core flow.

The results demonstrate strong evidence of equilibrium among the vibrational and rotational modes, particularly during the nominal test time and beyond. For all shots, at both nominal conditions (higher enthalpy and lower enthalpy), transient behavior in the temperature and partial pressure traces is observed during the first millisecond of nozzle flow. There appears to be a consistent upward trend in vibrational temperature during the first half-millisecond or so, followed shortly after by a slow relaxation toward the rotational temperature. The theoretical interpretation of this trend at early times is not yet clear, but may be attributed to the transient nature of the nozzle startup process while steady-state operation is being established. Similar to the trend in temperature, the partial pressure of NO decreases steadily across all $18 \mathrm{MJ} / \mathrm{kg}$ experiments. This is consistent with the undertailored nature of this condition, also evident in the nozzle reservoir pressure traces as described in Sec. IV. Future experiments will adjust this test condition to achieve a more closely tailored reservoir.

\section{Conclusions}

TDLAS was employed in preliminary efforts to characterize the freestream of the GALCIT T5 reflected shock tunnel facility operating under two nominal conditions [lower $(8 \mathrm{MJ} / \mathrm{kg})$ and higher (18 MJ $/ \mathrm{kg}$ ) stagnation enthalpy], each generating Mach 5 flows. The mid-IR laser diagnostics provided time-resolved measurements of NO absorbance from several NO rovibrational transitions, enabling sensitive estimation of the vibrational and rotational temperatures, as well as core flow path-averaged NO partial pressure. The data demonstrate a sizeable NO population and strongly indicate thermal equilibrium of this species during and after the test period (characterized by roughly static reservoir conditions). In addition to $\mathrm{NO}$, measurable quantities of $\mathrm{CO}$ and $\mathrm{H}_{2} \mathrm{O}$ were also observed, though their concentrations cannot be accurately estimated. Future experiments will improve upon these measurements by directly probing the homogenous core flow of the freestream (i.e., eliminating potential impacts from the boundary layer) with custom-built optical armatures, enabling a more quantitative characterization.

\section{Acknowledgments}

This work was supported by the Air Force Office of Scientific Research (AFOSR) through grant number FA9550-19-1-0219, with Ivett Leyva and Sarah Popkin as contract monitors. We would like to acknowledge the help of Bahram Valiferdowsi in running the experiments.

\section{References}

[1] Hank, J., Murphy, J., and Mutzman, R., "The X-51A Scramjet Engine Flight Demonstration Program," 15th AIAA International Space Planes and Hypersonic Systems and Technologies Conference, AIAA Paper 2008-2540, May 2008.

https://doi.org/10.2514/6.2008-2540

[2] Walker, S., Sherk, J., Shell, D., Schena, R., Bergmann, J., and Gladbach, J., "The DARPA/AF Falcon Program: The Hypersonic Technology Vehicle \#2 (HTV-2) Flight Demonstration Phase," 15th AIAA International Space Planes and Hypersonic Systems and Technologies Conference, Vol. 2, AIAA Paper 2008-2539, May 2008. https://doi.org/10.2514/6.2008-2539
[3] Dolvin, D., "Hypersonic International Flight Research and Experimentation Technology Development and Flight Certification Strategy," 16th AIAA/DLR/DGLR International Space Planes and Hypersonic Systems and Technologies Conference, AIAA Paper 2009-7228, 2009.

https://doi.org/10.2514/6.2009-7228

[4] Dolvin, D., "Hypersonic International Flight Research and Experimentation (HIFiRE) Fundamental Science and Technology Development Strategy," 16th AIAA/DLR/DGLR International Space Planes and Hypersonic Systems and Technologies Conference, AIAA Paper 2008-2581, 2008.

https://doi.org/10.2514/6.2008-2581

[5] Urzay, J., "Supersonic Combustion in Air-Breathing Propulsion Systems for Hypersonic Flight," Annual Review of Fluid Mechanics, Vol. 50, No. 1, 2018, pp. 593-627. https://doi.org/10.1146/annurev-fluid-122316-045217

[6] Li, Z., Sohn, I., and Levin, D. A., "Modeling of Nitrogen Monoxide Formation and Radiation in Nonequilibrium Hypersonic Flows," Journal of Thermophysics and Heat Transfer, Vol. 28, July 2014, pp. 365-380. https://doi.org/10.2514/1.T3940

[7] Bender, J. D., Valentini, P., Nompelis, I., Paukku, Y., Varga, Z., Truhlar, D. G., Schwartzentruber, T., and Candler, G. V., "An Improved Potential Energy Surface and Multi-Temperature Quasiclassical Trajectory Calculations of N2 + N2 Dissociation Reactions," Journal of Chemical Physics, Vol. 143, No. 5, 2015, Paper 054304. https://doi.org/10.1063/1.4927571

[8] Chaudhry, R. S., Grover, M. S., Bender, J. D., Schwartzentruber, T. E., and Candler, G. V., "Quasiclassical Trajectory Analysis of Oxygen Dissociation via $\mathrm{O}_{2}, \mathrm{O}$, and $\mathrm{N}_{2}$," AIAA Aerospace Sciences Meeting, AIAA Paper 2018-0237, 2018. https://doi.org/10.2514/6.2018-0237

[9] Valentini, P., Schwartzentruber, T. E., Bender, J. D., Nompelis, I., and Candler, G. V., "Direct Molecular Simulation of Nitrogen Dissociation Based on an ab initio Potential Energy Surface," Physics of Fluids, Vol. 27, No. 8, 2015, Paper 86102. https://doi.org/10.1063/1.4929394

[10] Wysong, I. J., and Gimelshein, S. F., "Hypersonic Non-Equilibrium Comparison Cases," AIP Conference Proceedings, Vol. 2132, No. 1, Aug. 2019.

https://doi.org/10.1063/1.5119603

[11] Bose, D., McCorkle, E., Thompson, C., Bogdanoff, D., Allen, G., and Grinstead, J., "Analysis and Model Validation of Shock Layer Radiation in Air," 46th AIAA Aerospace Sciences Meeting and Exhibit, AIAA Paper 2008-1246, 2008. https://doi.org/10.2514/6.2008-1246

[12] Nompelis, I., Candler, G., Holden, M., and Wadhams, T., "Computational Investigation of Hypersonic Viscous/Inviscid Interactions in High Enthalpy Flows," 36th AIAA Thermophysics Conference, AIAA Paper 2003-3642, 2003. https://doi.org/10.2514/6.2003-3642

[13] Karl, S., Hannemann, K., MacK, A., and Steelant, J., "CFD Analysis of the HyShot II Scramjet Experiments in the HEG Shock Tunnel," 15th AIAA International Space Planes and Hypersonic Systems and Technologies Conference, AIAA Paper 2008-2548, 2008. https://doi.org/10.2514/6.2008-2548

[14] Chaudhry, R., and Candler, G., "Computing Measured Spectra from Hypersonic Pitot Probes with Flow-Parallel Freestream Disturbances," AIAA Journal, Vol. 55, No. 12, Dec. 2017, pp. 4155-4166. https://doi.org/10.2514/1.J055396

[15] Kidd, C., and Adams, J., Jr., "Fast-Response Heat-Flux Sensor for Measurement Commonality in Hypersonic Wind Tunnels," Journal of Spacecraft and Rockets, Vol. 38, No. 5, Sept.-Oct. 2001, pp. 719-729. https://doi.org/10.2514/2.3738

[16] Loftus, A., and Krupenie, P. H., "The Spectrum of Molecular Nitrogen," Journal of Physical and Chemical Reference Data, Vol. 6, No. 1, 1977, p. 113. https://doi.org/10.1063/1.555546

[17] Krupenie, P. H., "The Spectrum of Molecular Oxygen," Journal of Physical and Chemical Reference Data, Vol. 1, No. 2, 1972, p. 423. https://doi.org/10.1063/1.3253101

[18] Shemansky, D. E., "N2 Vegard-Kaplan System in Absorption," Journal of Chemical Physics, Vol. 51, 1969, p. 689. https://doi.org/10.1063/1.1672058

[19] Brown, L. R., "Experimental Line Parameters of the Oxygen A Band at 760 nm," Journal of Molecular Spectroscopy, Vol. 199, No. 2, 2000, pp. 116-179. https://doi.org/10.1006/jmsp.1999.8012

[20] Danehy, P., O’Byrne, S., Houwing, A. F., Fox, J., and Smith, D., "FlowTagging Velocimetry for Hypersonic Flows Using Fluorescence of 
Nitric Oxide," AIAA Journal, Vol. 41, No. 2, Feb. 2003, pp. 263-271. https://doi.org/10.2514/2.1939

[21] Jiang, N., Webster, M., Lempert, W., Miller, J., Meyer, T., Ivey, C., and Danehy, P., "MHz-Rate Nitric Oxide Planar Laser-Induced Fluorescence Imaging in a Mach 10 Hypersonic Wind Tunnel," Applied Optics, Vol. 50, No. 4, 2011, pp. A20-A28. https://doi.org/10.1364/AO.50.000A20

[22] Lee, M. P., McMillin, B. K., Palmer, J. L., and Hanson, R. K., "Planar Fluorescence Imaging of a Transverse Jet in a Supersonic Crossflow," Journal of Propulsion and Power, Vol. 8, No. 4, 1992, pp. 729-735. https://doi.org/10.2514/3.23542

[23] McMillin, B. K., Palmer, J. L., and Hanson, R. K., "Temporally Resolved, Two-Line Fluorescence Imaging of NO Temperature in a Transverse Jet in a Supersonic Cross Flow," Applied Optics, Vol. 32, No. 36, 1993, pp. 7532-7545. https://doi.org/10.1364/ao.32.007532

[24] Mohamed, A. K., Henry, D., Faléni, J., Sagnier, P., Soutadé, J., Beck, W. H., and Schramm, J. M., "Infrared Diode Laser Absorption Spectroscopy Measurements in the S4MA, F4 and HEG Hypersonic Flows," International Symposium on Atmospheric Reentry Vehicles and Systems, AIAA Paper 1998-2870, March 1999. https://doi.org/10.2514/6.1998-2870

[25] MacDermott, W. N., and Marshall, J. C., "Nonequilibrium Nozzle Expansions of Partially Dissociated Air: A Comparison of the Theory and Electron-Beam Measurements," ARO INC Arnold Air Force Station Tennessee, AEDC-TR-69-66, 1969.

[26] Babikian, D. S., Gopaul, N., and Park, C., "Measurement and Analysis of Nitric Oxide Radiation in an Arc-Jet Flow," 28th AIAA Thermophysics Conference, Vol. 8, No. 4, Oct.-Dec. 1994, p. 737.

[27] Park, C., and Lee, S. H., "Validation of Multitemperature Nozzle Flow Code," Journal of Thermophysics and Heat Transfer, Vol. 9, No. 1, 1995, pp. 9-16.

[28] Parker, R., Wakeman, T., MacLean, M., and Holden, M., "Measuring Nitric Oxide Freestream Concentration Using Quantum Cascade Lasers at CUBRC," 44th AIAA Aerospace Science Meeting and Exhibit, AIAA Paper 2006-0926, 2006. https://doi.org/10.2514/6.2006-926

[29] Parker, R., Wakeman, T., MacLean, M., and Holden, M., "Measuring Nitric Oxide Freestream Velocity Using Quantum Cascade Lasers at CUBRC," 45th AIAA Aerospace Science Meeting and Exhibit, AIAA Paper 2007-1329, 2007 https://doi.org/10.2514/6.2007-1329

[30] Nompelis, I., and Candler, G., "Investigation of Hypersonic DoubleCone Flow Experiments at High Enthalpy in the LENS Facility," 45th AIAA Aerospace Science Meeting and Exhibit, AIAA Paper 2007-0203, 2007.

https://doi.org/10.2514/6.2007-203
[31] Parziale, N. J., Jewell, J. S., Shepherd, J. E., and Hornung, H. G., "Shock Tunnel Noise Measurement with Resonantly Enhanced Focused Schlieren Deflectometry," 28th International Symposium on Shock Waves, edited by K. Kontis, Springer, Berlin, 2012, pp. 747-752. https://doi.org/10.1007/978-3-642-25688-2_113

[32] Leyva, I., Laurence, S., Beierholm, A., Hornung, H., Wagnild, R., and Candler, G., "Transition Delay in Hypervelocity Boundary Layers by Means of $\mathrm{CO}_{2} /$ Acoustic Instability Interactions," 47th AIAA Aerospace Science Meeting, AIAA Paper 2009-1287, 2009.

https://doi.org/10.2514/6.2009-1287

[33] Hanson, R. K., Spearrin, R. M., and Goldenstein, C. S., Spectroscopy and Optical Diagnostics for Gases, Springer International Publ., Switzerland, 2016, Chaps. 7, 8. https://doi.org/10.1007/9783319232522

[34] Rothman, L. S., Gordon, I. E., Barber, R. J., Dothe, H., Gamache, R. R., Goldman, A., Perevalov, V., Tashkun, S. A., and Tennyson, J., "HITEMP, the High-Temperature Molecular Spectroscopic Database," Journal of Quantitative Spectroscopy and Radiative Transfer, Vol. 111, No. 15,2010 , pp. 2139-2150. https://doi.org/10.1016/j.jqsrt.2010.05.001

[35] Hargreaves, R. J., Gordon, I. E., Rothman, L. S., Tashkun, S. A., Perevalov, V. I., Lukashevskaya, A. A., Yurchenko, S. N., Tennyson, J., and Müller, H. S. P., "Spectroscopic Line Parameters of NO, $\mathrm{NO}_{2}$, and $\mathrm{N}_{2} \mathrm{O}$ for the HITEMP Database," Journal of Quantitative Spectroscopy and Radiative Transfer, Vol. 232, July 2019, pp. 35-53. https://doi.org/10.1016/j.jqsrt.2019.04.040

[36] Hornung, H., Sturtevant, B., Bélanger, J., Sanderson, S., Brouillette, M., and Jenkins, M., "Performance Data of the New Free-Piston Shock Tunnel at GALCIT," Shock Waves, Springer, Berlin, 1992, pp. 603 610. https://doi.org/10.1007/978-3-642-77648-9_95

[37] Parziale, N., "Slender-Body Hypervelocity Boundary-Layer Instability,” Ph.D. Thesis, California Inst. of Technology, California, 2013

[38] Candler, G. V., "Hypersonic Nozzle Analysis Using an Excluded Volume Equation of State," Proceedings of 38th AIAA Thermophysics Conference, AIAA Paper 2005-5202, 2005. https://doi.org/10.2514/6.2005-5202

[39] Wagnild, R. M., "High Enthalpy Effects on Two Boundary Layer Disturbances in Supersonic and Hypersonic Flow," Ph.D. Thesis, Univ. of Minnesota, Minneapolis, MN, 2012.

[40] Smith, G. P., Golden, D. M., Frenklach, M., Moriarty, N. W., Eiteneer, B., Golden-berg, M. C., Bowman, T., Hanson, R. K., Song, S., Gardiner, W. C., Jr., Lissianski, V. V., and Qin, Z., GRI-Mech 3.0, 1999, http:// combustion.berkeley.edu/gri-mech/version30/text30.html.

P. J. Drummond Associate Editor 